\title{
Atypical meningioma: a retrospective analysis of six cases and literature review
}

\author{
Yiming Liang ${ }^{1}$, Bo Ning ${ }^{1}$, Xing Hua ${ }^{2}$, Zhiping Liang ${ }^{3}$, Jingchao Ye ${ }^{1}$, Fangyi Yu ${ }^{1}$, Zhilei Xu ${ }^{1}$, Jiaxiang Chen ${ }^{1}$ \\ ${ }^{1}$ Department of Neurosurgery, Guangzhou Red Cross Hospital, The Fourth Affiliated Hospital of Jinan University, Guangzhou, China; ${ }^{2}$ Department \\ of Pathology, Guangzhou Red Cross Hospital, The Fourth Affiliated Hospital of Jinan University, Guangzhou, China; ${ }^{3}$ Department of Radiology, \\ Guangzhou Red Cross Hospital, The Fourth Affiliated Hospital of Jinan University, Guangzhou, China \\ Contributions: (I) Conception and design: Y Liang; (II) Administrative support: None; (III) Provision of study materials or patients: All authors; (IV) \\ Collection and assembly of data: Y Liang; (V) Data analysis and interpretation: Y Liang; (VI) Manuscript writing: All authors; (VII) Final approval of \\ manuscript: All authors. \\ Correspondence to: Yiming Liang. Department of Neurosurgery, Guangzhou Red Cross Hospital, The Fourth Affiliated Hospital of Jinan University, \\ Guangzhou 510220, China. Email: 471108118@qq.com.
}

Background: Atypical meningiomas (AM) are WHO grade II tumors with high heterogeneity and invasiveness, which are unique in their clinical presentation, imaging, pathology, treatment, and prognosis. In 2016, the diagnosis of AM remodified by the classification of central nervous system tumors of World Health Organization (WHO). In order to further analyze the diagnosis and treatment characteristics of AM, the clinical diagnosis, surgery treatment and follow-up data of 6 patients with AM in our hospital from January 2016 to December 2019 were analyzed retrospectively.

Methods: The clinical diagnosis, treatment and follow-up data of six patients with AM admitted to our hospital between January 2016 and December 2019 were analyzed retrospectively, and related literature was reviewed.

Results: The main clinical symptoms of the patients were increased intracranial pressure and functional area symptoms. MRI showed obvious enhancement, an unclear boundary with adjacent brain tissue, a large edema signal shadow in the white matter of peripheral brain tissue, and rapid enlargement in some cases. Pathological manifestations showed local necrosis and increased nuclear division activity. The results of immunocytochemistry showed that vimentin and epithelial membrane antigen (EMA) were positive, S-100 protein (S-100) and glial fibrillary acidic protein (GFAP) were negative, and the Ki-67 proliferation index was increased. Simpson's grade I-II resection was performed in five cases and no recurrence found after 4 to 46 months of follow-up. Simpson's grade IV resection was performed in one case and no follow-up was performed after discharge.

Conclusions: AMs are highly heterogeneous and invasive. Histologic diagnosis is the gold standard for diagnosis and surgical resection is the first treatment choice. Total resection (Simpson I-II) helps to reduce recurrence.

Keywords: Atypical meningioma (AM); diagnostic imaging; pathology; treatment, case series

Submitted Jan 08, 2021. Accepted for publication Mar 17, 2021.

doi: $10.21037 /$ tcr-21-375

View this article at: http://dx.doi.org/10.21037/tcr-21-375

\section{Introduction}

The diagnosis of atypical meningiomas (AMs) was remodified in 2016 by the Classification of Central Nervous System Tumors of the World Health Organization
(WHO) (1). The biological characteristics of AM are between benign meningiomas and anaplastic meningiomas. AMs are WHO grade II tumors with high heterogeneity and invasiveness, and which are unique in their clinical 
presentation, imaging, pathology, treatment, and prognosis. To further analyze the diagnosis and treatment characteristics of AM, the data of six patients with AM admitted to our hospital from January 2016 to December 2019 were retrospectively analyzed.

We present the following article in accordance with the AME Case Series Checklist (available at http://dx.doi. org/10.21037/tcr-21-375).

\section{Methods}

\section{Clinical information}

From January 2016 to December 2019, six patients with AMs confirmed by pathology were treated in our hospital, accounting for $25.0 \%(6 / 24)$ of meningioma operations in our department in the same period. The patient group comprised of three males and three females and the age of onset was 52-87 years. Craniocerebral MRI enhanced scans were routinely performed before surgery in all patients. According to the location of the tumor, corresponding craniotomy was performed, the degree of surgical resection was evaluated by Simpson grading of meningioma resection degree (2), and follow-up was implemented. This study was approved by the Medical Ethics Committee of Guangzhou Red Cross Hospital, The Fourth Affiliated Hospital of Jinan University (2019-146-01). All procedures performed in this study involving human participants were in accordance with the Declaration of Helsinki (as revised in 2013). Individual consent for this retrospective analysis was waived.

\section{Histopathological examination}

The surgical specimens were diagnosed as AM according to the 2016 histological criteria of the WHO (1). Tumor tissues were all embedded in paraffin, stained with $\mathrm{HE}$, and observed under light microscope. A pathological result that meets any of the (I) to (III) criteria can be diagnosed as a WHO grade II AM: (I) Increased mitotic images (mitotic count $\geq 4 / 10 \mathrm{HPF}$ ); (II) invasion of brain parenchyma; (III) at least three of the following criteria: (i) cell density increased; (ii) small cell areas with high ratio of nucleus to cytoplasm appeared; (iii) distinct nucleolus; (iv) sheet distribution of tumor cells (swirling or bundle-like arrangement structure lost); (v) focal spontaneous necrosis (non-iatrogenic). Immunohistochemistry was used to detect the tumor-specific markers vimentin, epithelial membrane antigen (EMA), S-100 protein (S-100), glial fibrillary acidic protein (GFAP), and Ki67. The SP kit was purchased from Fuzhou Maixin Biotechnology Co., Ltd.

\section{Results}

\section{Magnetic resonance imaging and pathological manifestations}

The main clinical symptoms in the six patients were increased intracranial pressure (headache) and functional area symptoms such as alalia, limb weakness, and hearing loss (Table 1). Among the six cases, four tumor were located in the convex surface of the brain, (including one case in the right parietal region and invaded the adjacent skull bone; one case of a huge space in the right temporal region not only destroyed the right orbit and sphenoid bone, but also communicated to the infratemporal fossa), a further case was in the petroclival region of the skull base, and another case was found brain parenchyma invasion.

All patients underwent MRI and pathological examination. The MRI findings of 6 patients with atypical meningioma were mixed signal of equal/long T1 and long T2. In contrast-enhanced scan, there were obvious homogeneous or heterogeneous enhancement, unclear boundary with adjacent brain tissue, and large edema signal shadow in peripheral white matter. In some cases, preoperative MRI showed that the tumor increased rapidly. MR single voxel hydrogen spectrum analysis showed that Cho and Cho/NAA ratio increased in the focal area of the nodule.

Pathological HE staining largely showed the following features: the arrangement of tumor cells has lost the swirllike structure, the cell density is high, the nucleolus is obvious, and the focus of necrosis can be seen in the local area. The mitotic activity was increased ( $>4 / 10 \mathrm{HPF})$. The results of immunocytochemistry examination showed that vimentin was positive in all six cases, EMA was positive in four, S-100 was negative in four, GFAP was negative in all cases, and the Ki-67 proliferation index (hot spot region $>15 \%$ ) was found in five cases.

In case 1, MRI (Figure 1A) showed the left parietal lobe mass is inhomogeneously enhanced, with obvious edema; Pathological HE staining (Figure 1B) showed that the tumor cells are arranged in a lobular or nest-like shape, with vortex-like structure in the local area, the tumor nucleus is of medium-size, mitotic image was $(>4 / 10 \mathrm{HPF})$, and necrosis was seen in the local area.

In case 2, MRI (Figure 2A) showed mixed signal shadow 
Table 1 Clinical data of sic cases with atypical meningioma

\begin{tabular}{|c|c|c|c|c|c|c|c|c|}
\hline Number & Gender & Age & Lesion location & Symptom & MRI manifestations & HE staining & Immunohistochemistry & $\begin{array}{l}\text { Surgical results } \\
\text { and follow-up }\end{array}$ \\
\hline 1 & Female & 87 & $\begin{array}{l}\text { The left parietal } \\
\text { lobe }\end{array}$ & $\begin{array}{l}\text { Numbness } \\
\text { in right } \\
\text { extremities }\end{array}$ & $\begin{array}{l}\text { The size of tumor: } 5.1 \mathrm{~cm} \\
\times 5.8 \mathrm{~cm} \times 4.6 \mathrm{~cm} \text {, edema } \\
\text { was obvious with obvious } \\
\text { enlargement of lesion. Low } \\
\text { signal was shown in ADC }\end{array}$ & $\begin{array}{l}\text { Mitotic figures } \\
\text { (>4/10 HPF); } \\
\text { visible necrosis } \\
\text { foci }\end{array}$ & $\begin{array}{l}\text { Vimentin (+), EMA (+), } \\
\text { S-100 (-), GFAP }(-) \text {, } \\
\text { Ki67 }(20 \%+)\end{array}$ & $\begin{array}{l}\text { Simpson grade } \\
\text { I, followed } \\
\text { up for } \\
18 \text { months } \\
\text { without } \\
\text { recurrence }\end{array}$ \\
\hline 4 & Male & 67 & Petroclival area & $\begin{array}{l}\text { Tinnitus } \\
\text { with } \\
\text { hearing } \\
\text { loss }\end{array}$ & $\begin{array}{l}\text { The size of tumor: } \\
4.5 \mathrm{~cm} \times 3.6 \mathrm{~cm} \times 1.9 \mathrm{~cm} \text {, } \\
\text { enhanced scanning } \\
\text { lesions were significantly } \\
\text { enhanced. Low signal was } \\
\text { shown in ADC }\end{array}$ & $\begin{array}{l}\text { Increased cell } \\
\text { density; obvious } \\
\text { nucleolus, } \\
\text { nucleus deep } \\
\text { staining, visible } \\
\text { karyokinesis and } \\
\text { necrosis }\end{array}$ & $\begin{array}{l}\text { Vimentin (+), EMA (+), } \\
\text { S-100 (-), GFAP (-), } \\
\text { Ki67 (hot spot region } \\
5 \%+\text { or so) }\end{array}$ & $\begin{array}{l}\text { Simpson grade } \\
\text { II, followed } \\
\text { up for } \\
12 \text { months } \\
\text { without } \\
\text { recurrence }\end{array}$ \\
\hline 6 & Female & 52 & $\begin{array}{l}\text { The right } \\
\text { occipitoparietal } \\
\text { part }\end{array}$ & Headache & $\begin{array}{l}\text { The size of tumor: } \\
4.9 \mathrm{~cm} \times 4.8 \mathrm{~cm} \times 5.4 \mathrm{~cm} \text {, } \\
\text { with clear boundary, } \\
\text { obvious homogeneous } \\
\text { enhancement and sheet } \\
\text { edema. Bone erosion was } \\
\text { seen near the inner plate } \\
\text { of parietal bone. Equal and } \\
\text { slightly high signal were } \\
\text { shown in ADC }\end{array}$ & $\begin{array}{l}\text { Tumor cells } \\
\text { were arranged in } \\
\text { flake, bundle or } \\
\text { whirlpool shape } \\
\text { with obvious } \\
\text { nucleolus and } \\
\text { visible mitosis. } \\
\text { Some tumor } \\
\text { cells had small } \\
\text { nucleoli }\end{array}$ & $\begin{array}{l}\text { Vimentin (+), EMA (-), } \\
\text { S-100 (+/-), GFAP (-), } \\
\text { Ki67 (hot spot region } \\
7 \%+\text { or so) }\end{array}$ & $\begin{array}{l}\text { Simpson grade } \\
\text { I, followed } \\
\text { up for } \\
4 \text { months } \\
\text { without } \\
\text { recurrence }\end{array}$ \\
\hline
\end{tabular}

ADC, apparent diffusion coefficient; Cho/NAA, choline/N-acetylaspartate; EMA, epithelial membrane antigen; GFAP, glial fibrillary acidic protein; HPF, high power field; Ki67, Ki-67 proliferation index; S-100, S-100 protein. 

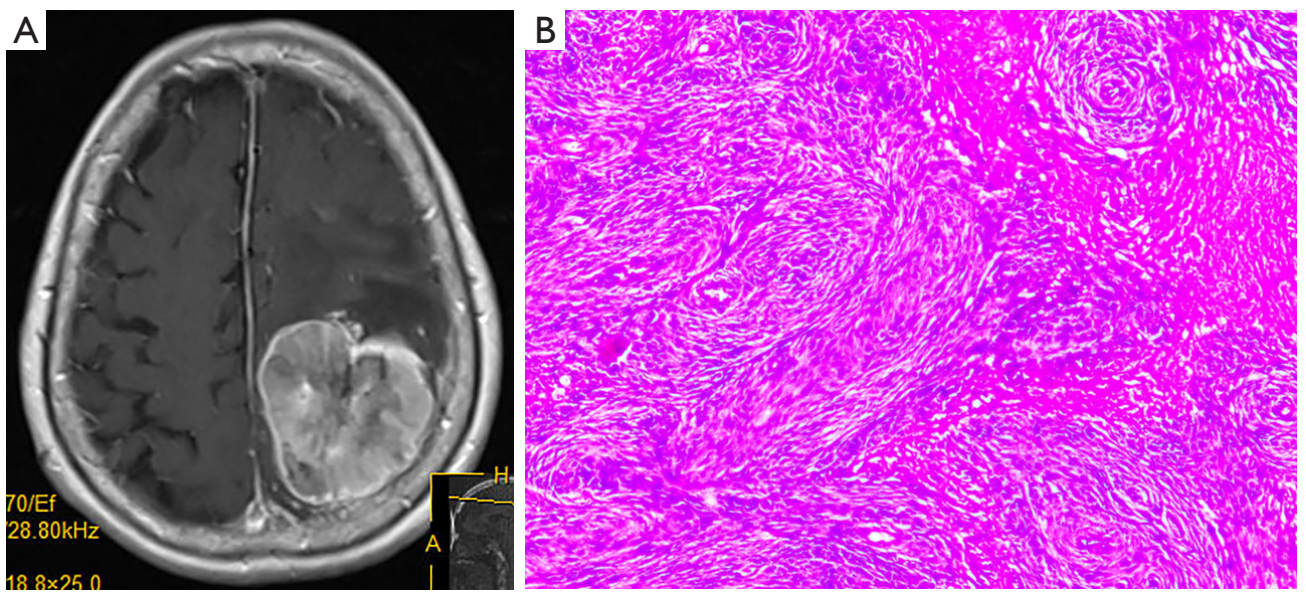

Figure $1 \mathrm{MRI}$ and pathological features of atypical meningiomas in clinical case 1. Female patient, 87 years old. (A) MRI shows the left parietal lobe mass is inhomogeneously enhanced, with obvious edema; (B) HE staining $(\times 100)$ shows that the tumor cells are arranged in a lobular or nest-like shape, with vortex-like structure in the local area, the tumor nucleus is of medium-size, mitotic image is $(>4 / 10 \mathrm{HPF})$, and necrosis is seen in the local area. MRI, magnetic resonance imaging; HE, hematoxylin-eosin.
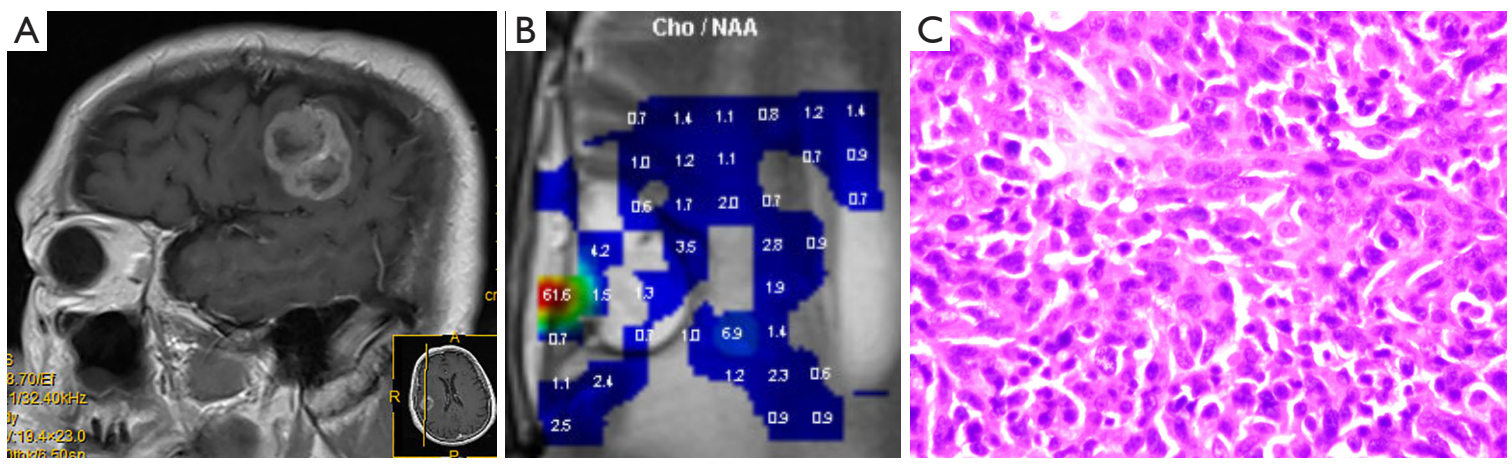

Figure 2 MRI and pathological features of atypical meningiomas in clinical case 2. Male patient, 71 years old. (A) MRI shows mixed signal shadow in the right frontal lobe, with ring enhancement. There are flake like non enhancement areas in the lesion and large edema of the white matter around it. (B) MR single voxel hydrogen spectrum analysis: the focus area of the right frontal lobe nodule: Cho is increased, the ratio of Cho/NAA is increased, and the spectrum shape of the lesion edge is generally normal. (C) HE staining ( $\times 200)$ showing tumor cells arranged in nest-like and whirlpool-like shapes, nuclei are of medium-size, some of the nuclei are vacuolated, mitotic images are common, and necrosis is seen in local areas. MRI, magnetic resonance imaging; MR, magnetic resonance; Cho/NAA, choline/N-acetylaspartate; HE, hematoxylin-eosin.

in the right frontal lobe, with ring enhancement, and large edema of the white matter. MR single voxel hydrogen spectrum analysis (Figure $2 B$ ) showed the focus area of the right frontal lobe nodule: Cho was increased, the ratio of Cho/NAA was increased, and the spectrum shape of the lesion edge was generally normal. HE staining (Figure 2C) showed tumor cells arranged in nest-like and whirlpool-like shapes, nuclei were of medium-size, some of the nuclei were vacuolated, mitotic images weree common, and necrosis was seen.
In case 3, MRI (Figure 3A) showed a huge tumor with rich blood supply in the right temporal lobe, which had invaded the lateral wall of the right orbit causing sphenoid bone damage, and invasion of the infratemporal fossa, pterygopalatine fossa, and medial pterygoid and lateral pterygoid muscles outside the cranium. HE staining (Figure 3B) showed tumor cells were arranged in small sheets, of papillary or whirlpool shape, with obvious nucleoli and abundant cytoplasm; Immunohistochemistry results show EMA (+/-), Vimentin (+), S100 (-), GFAP (-). 

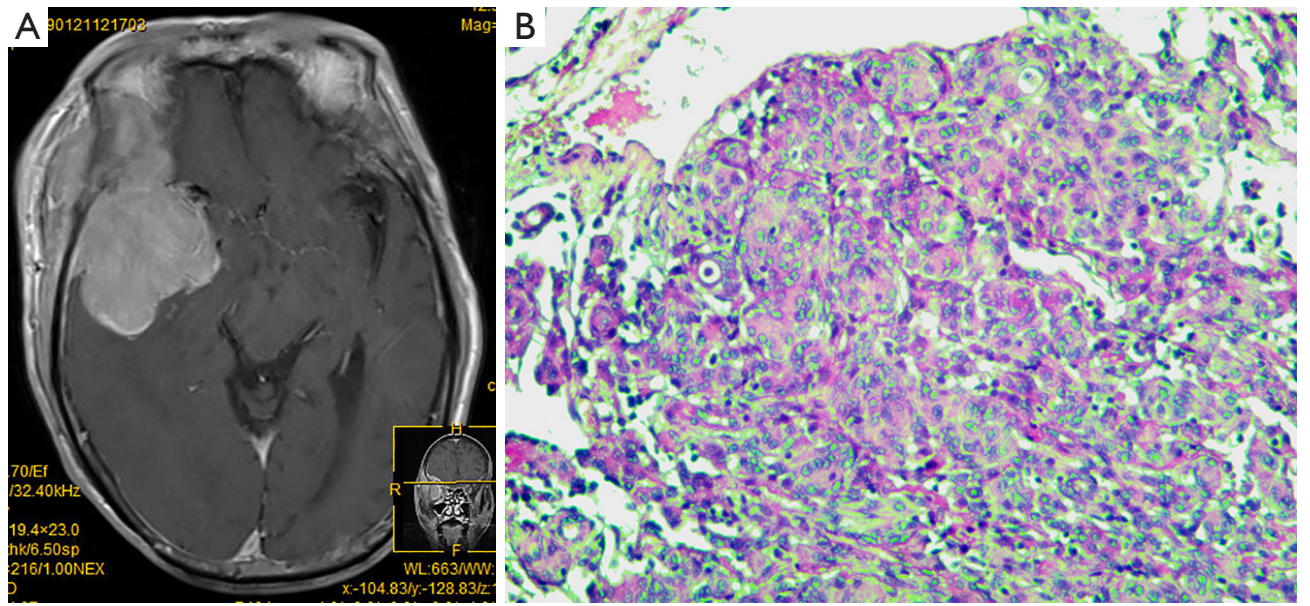

Figure $3 \mathrm{MRI}$ and pathological features of atypical meningiomas in clinical case 3. Male patient, 70 years old. (A) MRI shows a huge tumor with rich blood supply in the right temporal lobe, which has invaded the lateral wall of the right orbit causing sphenoid bone damage, and invasion of the infratemporal fossa, pterygopalatine fossa, and medial pterygoid and lateral pterygoid muscles outside the cranium. (B) HE staining $(\times 100)$ shows tumor cells are arranged in small sheets, of papillary or whirlpool shape, with obvious nucleoli and abundant cytoplasm; Immunohistochemistry results show EMA (+/-), Vimentin (+), S100 (-), GFAP (-). MRI, magnetic resonance imaging; HE, hematoxylin-eosin; EMA, epithelial membrane antigen; S-100: S-100 protein; GFAP, glial fibrillary acidic protein.
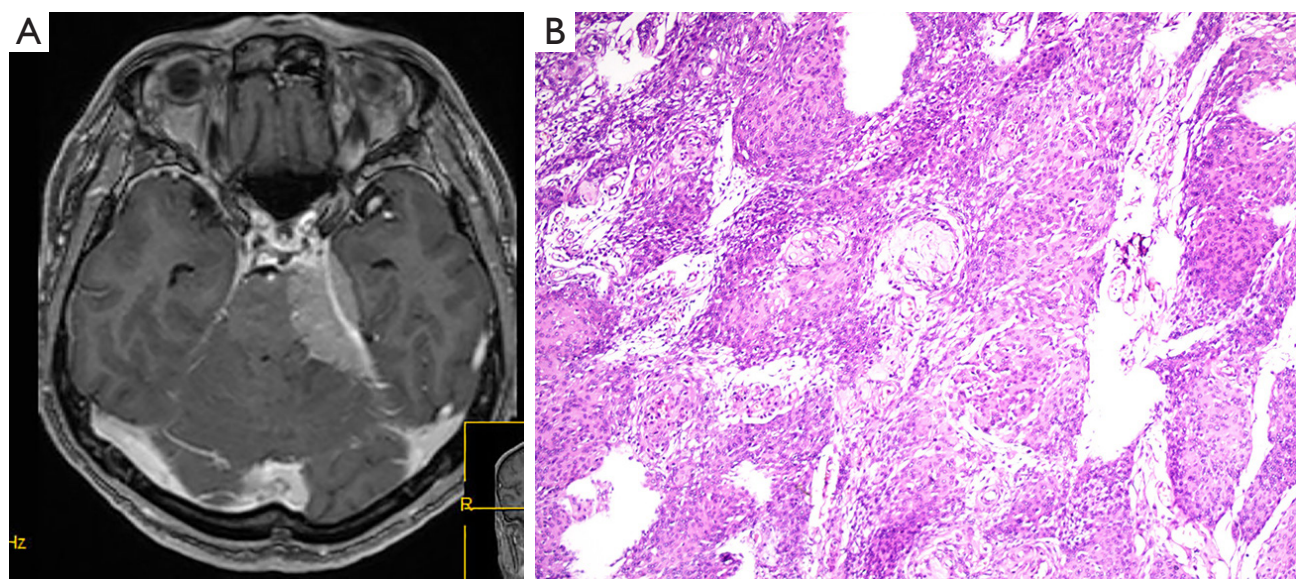

Figure 4 MRI and pathological features of atypical meningiomas in clinical case 4. Male patient, 67 years old. (A) MRI shows that T1 is slightly longer and the T2 signal is abnormal with irregular spindle shape in the left petroclival area, which connected with the left cerebellar tentorium by a wide base, grows across the tentorium, spreading forward into the middle cranial fossa and Meckel cavity, with obvious enhancement. (B) HE staining ( $\times 100)$ shows obvious nucleolus, deep staining, rough chromatin, mitosis, and necrosis. MRI, magnetic resonance imaging; HE, hematoxylin-eosin.

In case 4, MRI (Figure 4A) showed T1 signal was slightly longer and the T2 signal was abnormal with irregular spindle shape in the left petroclival area, which connected with the left cerebellar tentorium by a wide base, growed across the tentorium, spreading forward into the middle cranial fossa and Meckel cavity, with obvious enhancement.
HE staining (Figure 4B) showed obvious nucleolus, deep staining, rough chromatin, mitosis, and necrosis.

In case 5, MRI (Figure 5A) showed mass mixed signal shadow of the right frontotemporal part, equal T1/slightly long T2 signal, and enhanced scanning had obvious enhancement. HE staining (Figure 5B) showed that the 

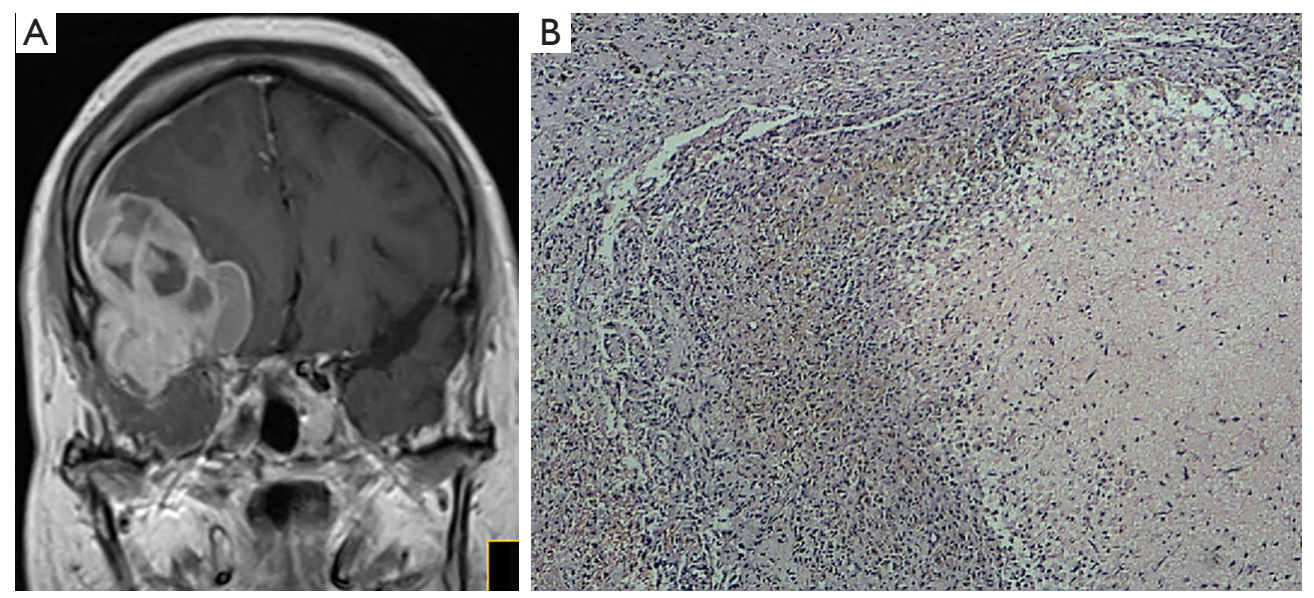

Figure $5 \mathrm{MRI}$ and pathological features of atypical meningiomas in clinical case 5. Female patient, 61 years old. (A) MRI shows mass mixed signal shadow of the right frontotemporal part, equal T1/slightly long T2 signal, and enhanced scanning had obvious enhancement. (B) HE staining $(\times 100)$ shows that the tumor cells are arranged in a lobular or nest-like shape, with vortex-like structure in the local area, and the tumor nucleus is of medium-size. The mitotic image is (>4/10 HPF). The tumor cell proliferation index is high (Ki-67 65\%). MRI, magnetic resonance imaging; HE, hematoxylin-eosin.

tumor cells were arranged in a lobular or nest-like shape, with vortex-like structure in the local area, and the tumor nucleus was of medium-size. The mitotic image was $(>4 / 10 \mathrm{HPF})$. The tumor cell proliferation index was high (Ki-67 65\%).

In case 6, MRI (Figure 6A) showed a round soft tissue like mass was under the inner plate of the right occipitoparietal part with clear boundary and obvious homogeneous enhancement. HE staining (Figure 6B) showed that the fusiform tumor cells were arranged in a flake, bundle or whirlpool shape, with an unclear cell boundary. Nuclei were long fusiform and fat fusiform. Some tumor cells had small nucleoli and mitosis.

\section{Surgical results and follow-up}

The degree of surgical resection of meningioma was evaluated according to the Simpson grade. Simpson grade I-II resection was performed in five cases, and there was no recurrence after follow-up for 4 to 46 months. However, one patient with right frontal lobe intracerebral occupancy developed a new metastatic tumor in the ipsilateral temporal occipital lobe 1 year after surgery. The patient's family refused further treatment. Simpson grade IV resection was performed in another case where a huge occupancy in the right temporal region destroyed the right orbit and sphenoid bone and communicated to the infratemporal fossa, and the follow-up was lost after discharge (Table 1).

\section{Discussion}

According to the WHO 2016 histological standards, AM is defined as WHO grade II, placing it between benign meningioma (WHO grade I) and anaplastic meningioma (WHO grade III). Grade II and III meningiomas were more common in men. The median age of diagnosis in Grade II and III was 57 years old, which were younger than grade I (3).

MRI is currently the most used imaging examination method to diagnose meningioma, and meningioma can be evaluated by parameters such as T1 and T2 weighting, diffusion weighting, and the apparent diffusion coefficient (ADC). It is generally believed that the following MRI features are helpful in the preoperative diagnosis of AMs (4-7): (I) the signal is mostly uneven, and the enhanced scan shows obvious uniform enhancement or uneven enhancement; (II) the edges are mostly irregular, mushroom-shaped, lobulated, and the boundary with the adjacent brain tissue is not clear; (III) large areas of edema signal shadow are found around the focal area; (IV) the volume is generally large, or the tumor increases obviously in a short time. The AMs in the current study largely exhibited these MRI imaging features.

Mere imaging manifestations do not have sufficient specificity to provide a definitive diagnosis. Although it is considered that obvious edema, lack of calcification, bone destruction, and cystic degeneration are the 

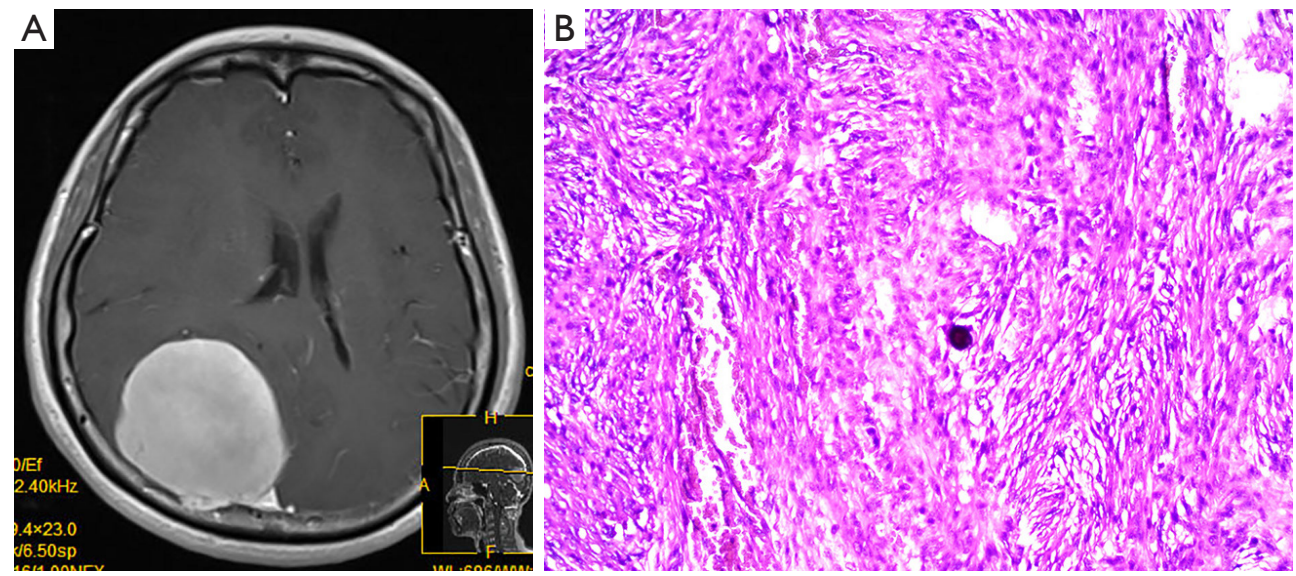

Figure 6 MRI and pathological features of atypical meningiomas in clinical case 6. Female, 52 years old. (A) MRI shows that a round soft tissue like mass was under the inner plate of the right occipitoparietal part with clear boundary and obvious homogeneous enhancement. (B) HE staining $(\times 100)$ shows that the fusiform tumor cells are arranged in a flake, bundle or whirlpool shape, with an unclear cell boundary. Nuclei are long fusiform and fat fusiform. Some tumor cells have small nucleoli and mitosis. MRI, magnetic resonance imaging; HE, hematoxylin-eosin.

characteristic features of malignant meningiomas, many benign meningiomas also have similar features. Some studies have even suggested that cystic and necrotic areas, uneven enhancement, and size, are not related to malignant meningiomas (5). It has been reported that a decrease of the $\mathrm{ADC}$ is a common MRI manifestation of AM. The ADC value is inversely proportional to the $\mathrm{Ki}-67$ proliferation index, which helps distinguish low-grade and aggressive meningiomas (6). Histologically, AMs create mitotic activity, necrosis, and the ratio of nucleus to cytoplasm and tumor cells increases, resulting in decreased water diffusion rates and lower $\mathrm{ADC}$ values.

In the cases in our study, one patient with right frontal intracerebral occupancy underwent MR single voxel hydrogen spectrum analysis. The results showed that the Cho increased in the focal area of the nodule, the Cho/NAA ratio elevated, and the spectral morphology of the edge of the lesion was almost normal. Previous studies have shown that on the magnetic resonance spectra, high $\mathrm{Cho/Cr}$ ratios and low NAA peaks are more likely to show atypical and anaplastic meningioma, and spectral features may help distinguish meningiomas from atypical types $(6,7)$.

Pathological examination is required to confirm the diagnosis of AM. A major modification to the 2016 WHO classification lists brain invasion as one of the diagnostic criteria for AM. Brain parenchyma encroachment is clearly defined as a condition in which tumor cells infiltrate brain parenchyma in an irregular and tongue-like shape without cerebral pia mater intervention. Previously, brain invasion was only recommended as prognostically considered WHO grade IIon was only recommended as s a condition in which meningioma (1).

The growth mode of AM is mainly infiltrative growth. During tumor growth, the surrounding arachnoid and cerebral pia mater are gradually destroyed, and the tumor cells further invade brain tissue (8). Brain invasion, a high mitotic index, and sheet distribution of tumor cells are significantly associated with shorter disease free survival (DFS) and have a higher risk of meningioma recurrence. In the absence of brain invasion or a high mitotic index, the recurrence rate of $A M$ is low (9). At present, the routine pathological diagnosis of AM is controversial because of inter-observer differences. One study compared the consistency of inter-observer findings in the evaluation of diagnostic criteria for AM. The results showed that spontaneous necrosis had the highest inter-observer consistency, while the consistency of flake area, high cellularity, and small cells were moderate (10).

Immunocytochemistry staining is a necessary auxiliary means for pathological diagnosis, which can distinguish AM from gliomas, neurogenic tumors, mesenchymal tumors, and some metastatic tumors. Both EMA and vimentin are the preferred auxiliary diagnostic markers for meningioma $(11,12)$. EMA is a specific marker of epithelial tissues. Most meningiomas express EMA, and the expression index decreases in high-risk meningiomas (WHO grade II, grade 
III). Vimentin is a specific marker of mesenchymal tissue. It is positive in almost all meningiomas, especially in high-risk meningioma (WHO grade II, III) and the expression index rises, showing diffuse positivity. S-100 and GFAP are both acidic proteins distributed in the nervous system. A finding of negative S-100 and negative GFAP can usually rule out neuroepithelial tumors. The main immunocytochemical results of the six cases of AM in this group showed vimentin to be positive in all cases and GFAP to be negative in all cases, and EMA to be positive and S-100 to be negative in four of the six cases. These findings are consistent with the literature.

The Ki67 proliferation index was increased in 5 of the 6 cases (hot spot region $>5 \%$ ). Ki-67 rises with the increase of tumor malignancy degree, with an average of $3.8 \%$ for benign meningiomas and $7.2 \%$ for AMs. AMs with high expression of $\mathrm{Ki}-67$ were prone to relapse after surgery (13). Barrett et al. reported that among many pathological indexes including brain invasion, increased cell density, obvious nucleolus, sheet distribution of tumor cells, mitotic number, and Ki67 proliferation index, only the Ki67 proliferation index and mitosis number could predict local recurrence (14). However, due to the small number of cases and the short follow-up time in our study, we failed to observe the relation between high Ki-67 expression and poor prognosis.

Although histological diagnosis and grading remains the current gold standard for diagnosing meningioma, molecular techniques are increasingly used for diagnosing meningioma pathogenesis, development, and the hypothesis of treatment and diagnosis. Studies have confirmed that NF2 mutations and/or chromosome 22 deletions play a key role in the occurrence of AMs $(15,16)$ and it has been suggested that DNA methylation profile can stratify tumor behavior more accurately (17). Molecular characterization of tumors based on gene mutations such as NF2, SMO, TERT, TRAF7, and methylation profiles is under development. Future developments may see molecular techniques combined with histology used to classify meningiomas and guide individual targeted therapy $(3,15-17)$.

While surgical resection remains the first choice for the treatment of AM, there are two types of meningiomas that are difficult to treat surgically. The first of these is seen when the lesion, even those which are low-grade, surrounds vascular and neural structures, and removal carries major risks and is likely to cause functional damage. The second involves Grade II-III meningiomas located on the convex surface of the skull as these frequently relapse, especially when performing invasive venous sinus obstruction surgery for total resection of the meningioma. The goal of the operation in this case is to completely remove the tumor and its involved dura mater and skull. For AMs, the tumor should be removed as completely as possible, along with the dura mater at the base of the tumor and even the invaded skull (Simpson I-II grade) (18).

The degree of surgical resection was a major factor affecting the prognosis of patients and total resection was identified as an independent prognostic factor for the survival by Champeaux et al. (19). Total resection of AMs results in a longer overall survival and progressionfree survival (20-22). In our study, complete resection was performed in five cases (Simpson I-II grade) and there was no recurrence after follow-up for 4 to 46 months. However, more cases and longer follow-up time are needed to further evaluate outcomes. Adjuvant radiotherapy for patients with total resection of AM is still controversial (23). In one study, the recurrence rates of patients without adjuvant radiotherapy after total resection were $7 \%$ in 1 year, $41 \%$ in 5 years, and $48 \%$ in 10 years (24). Patients who failed to have complete resection had a high rate of recurrence and in these cases postoperative adjuvant radiotherapy is usually recommended. Clinical trials such as EORTC 22042 (NCT 00626730), RTOG 0539 (NCT 00895622), and ROAM/ EORTC-1308 have been used to investigate the role of radiotherapy in the treatment of patients with meningioma at WHO grade II and grade III $(3,25)$. A study has also recommended that postoperative treatment be limited to meningiomas with high mitoses, sheet distribution of tumor cells, or brain invasion (9).

$\mathrm{AM}$ is a highly heterogeneous and invasive tumor, and its imaging findings are not specific. Histological diagnosis and grading remain the gold standard of diagnosis, but molecular technique diagnosis will more accurately stratify the behavior of the tumor. Surgical resection is still the first choice for the treatment of AMs and total resection (Simpson I-II grade) should be performed to reduce recurrence.

\section{Acknowledgments}

Funding: None.

\section{Footnote}

Reporting Checklist: The authors have completed the 
AME Case Series Checklist. Available at http://dx.doi. org/10.21037/tcr-21-375

Data Sharing Statement: Available at http://dx.doi. org/10.21037/tcr-21-375

Conflicts of Interest: All authors have completed the ICMJE uniform disclosure form (available at http://dx.doi. org/10.21037/tcr-21-375). The authors have no conflicts of interest to declare.

Ethical Statement: The authors are accountable for all aspects of the work in ensuring that questions related to the accuracy or integrity of any part of the work are appropriately investigated and resolved. All procedures performed in this study involving human participants were in accordance with the Declaration of Helsinki (as revised in 2013). This study was approved by the Medical Ethics Committee of Guangzhou Red Cross Hospital, The Fourth Affiliated Hospital of Jinan University (2019-146-01). Individual consent for this retrospective analysis was waived.

Open Access Statement: This is an Open Access article distributed in accordance with the Creative Commons Attribution-NonCommercial-NoDerivs 4.0 International License (CC BY-NC-ND 4.0), which permits the noncommercial replication and distribution of the article with the strict proviso that no changes or edits are made and the original work is properly cited (including links to both the formal publication through the relevant DOI and the license). See: https://creativecommons.org/licenses/by-nc-nd/4.0/.

\section{References}

1. Louis DN, Perry A, Reifenberger G, et al. The 2016 World Health Organization Classification of Tumors of the Central Nervous System: a summary. Acta Neuropathologica 2016;131:803-20.

2. Simpson D. The recurrence of intracranial meningiomas after surgical treatment. J Neurol Neurosurg Psychiatry 1957;20:22-39.

3. Apra C, Peyre M, Kalamarides M. Current treatment options for meningioma. Expert Rev Neurother 2018;18:241-9.

4. Surov A, Gottschling S, Mawrin C, et al. DiffusionWeighted Imaging in Meningioma: Prediction of Tumor Grade and Association with Histopathological Parameters. Transl Oncol 2015;8:517-23.
5. Nagar VA, Ye JR, Ng WH, et al. Diffusion-weighted MR imaging: diagnosing atypical or malignant meningiomas and detecting tumor dedifferentiation. AJNR Am J Neuroradiol 2008;29:1147-52.

6. Messerer M, Richoz B, Cossu G, et al. Recent advances in the management of atypical meningiomas. Neurochirurgie 2016;62:213-22.

7. Tan LA, Boco T, Johnson AK, et al. Magnetic resonance imaging characteristics of typical and atypical/anaplastic meningiomas - Case series and literature review. Br J Neurosurg 2015;29:77-81.

8. Savary C, Rousselet MC, Michalak S, et al. Solitary fibrous tumors and hemangiopericytomas of the meninges: Immunophenotype and histoprognosis in a series of 17 cases. Ann Pathol 2016;36:258-67.

9. Barresi V, Lionti S, Caliri S, et al. Histopathological features to define atypical meningioma: What does really matter for prognosis? Brain Tumor Pathol 2018;35:168-80.

10. Rogers CL, Perry A, Pugh S, et al. Pathology concordance levels for meningioma classification and grading in NRG Oncology RTOG Trial 0539. Neuro Oncol 2016;18:565-74.

11. Rogers L, Barani I, Chamberlain M, et al. Meningiomas: knowledge base, treatment outcomes, and uncertainties. A RANO review. J Neurosurg 2015;122:4-23.

12. Wen M, Jung S, Moon KS, et al. Immunohistochemical profile of the dural tail in intracranial meningiomas. Acta Neurochir (Wien) 2014;156:2263-73.

13. Devaprasath A, Chacko G. Diagnostic validity of the Ki-67 labeling index using the MIB-1 monoclonal antibody in the grading of meningiomas. Neurol India 2003;51:336-40.

14. Barrett OC, Hackney JR, McDonald AM, et al. Pathologic Predictors of Local Recurrence in Atypical Meningiomas Following Gross Total Resection. Int J Radiat Oncol Biol Phys 2019;103:453-9.

15. Yuzawa S, Nishihara H, Tanaka S. Genetic landscape of meningioma. Brain Tumor Pathol 2016;33:237-47.

16. Clark VE, Erson-Omay EZ, Serin A, et al. Genomic analysis of non-NF2 meningiomas reveals mutations in TRAF7, KLF4, AKT1, and SMO. Science 2013;339:1077-80.

17. Sahm F, Schrimpf D, Stichel D, et al. DNA methylationbased classification and grading system for meningioma: a multicentre, retrospective analysis. Lancet Oncol 2017;18:682-94.

18. Paldor I, Awad M, Sufaro YZ, et al. Review of 
controversies in management of non-benign meningioma. J Clin Neurosci 2016;31:37-46.

19. Champeaux C, Jecko V, Houston D, et al. Malignant Meningioma: An International Multicentre Retrospective Study. Neurosurgery 2019;85:E461-69.

20. Durand A, Labrousse F, Jouvet A, et al. WHO grade II and III meningiomas: a study of prognostic factors. J Neurooncol 2009;95:367-75.

21. Sughrue ME, Kane AJ, Shangari G, et al. The relevance of Simpson Grade I and II resection in modern neurosurgical treatment of World Health Organization Grade I meningiomas. J Neurosurg 2010;113:1029-35.

22. Zaher A, Abdelbari MM, Zayed DH, et al. Atypical meningioma: a study of prognostic factors. World

Cite this article as: Liang $\mathrm{Y}$, Ning $\mathrm{B}$, Hua $\mathrm{X}$, Liang Z, Ye J, Yu F, Xu Z, Chen J. Atypical meningioma: a retrospective analysis of six cases and literature review. Transl Cancer Res 2021;10(3):1509-1518. doi: 10.21037/tcr-21-375
Neurosurg 2013;80:549-53.

23. Zhi M, Girvigian MR, Miller MJ, et al. Long-Term Outcomes of Newly Diagnosed Resected Atypical Meningiomas and the Role of Adjuvant Radiotherapy. World Neurosurg 2019;122:e1153-61.

24. Aghi MK, Carter BS, Cosgrove GR, et al. Long-term recurrence rates of atypical meningiomas after gross total resection with or without postoperative adjuvant radiation. Neurosurgery 2009;64:56-60, 60.

25. Jenkinson MD, Santarius T, Zadeh G, et al. Atypical meningioma-is it time to standardize surgical sampling techniques? Neuro Oncol 2017;19:453-4.

(English Language Editor: B. Draper) 